\title{
Electromyographic Silent Periods and Jaw Motion Parameters: Quantitative Measures of Temporomandibular Joint Dysfunction
}

\author{
J. O. Bailey, JR., W. D. MaCall, JR., and M. M. Ash, JR. \\ Stomatognathic Laboratory, Department of Occlusion, Dental Research Institute, School of \\ Dentistry, University of Michigan, Ann Arbor, Michigan 48109, USA
}

The purpose of this research was to compare, in the subjects, the duration of the EMG silent: period with jaw motion error. The results indicate that both jaw motion error and silent period duration are large in patients with TMJmuscle-pain dysfunction, both are small in normal subjects, and both are small in successfully treated patients. There is a statistically significant correlation $(r=0.91 ; \mathrm{P}<0.01)$ between the two diagnostic parameters of $T M J$-musclepain dysfunction.

Electromyography has been used to evaluate patients with TMJ-muscle-pain-dysfunction..$^{1,2}$ These reports suggested that the duration of the silent period following a tap to the menton during a maximal clench is a valid measure of such dysfunction. However, the silent period duration has not been tested against any other measure of TMJ dysfunction such as jaw motion error. ${ }^{3}$

A need exists in clinical dentistry and research to quantify the symptoms and dysfunction associated with TMJ-muscle-pain disturbances. The numerous epidemiological aspects have been reasonably documented ${ }^{4-6}$ but the significance of the disease in respect to the need for treatment, the effectiveness of treatment, and the economics of the problem have not been reported. Such investigations would be facilitated by quantitative parameters such as those described here.

The dimensions of disagreement in clinical dental research extend from the nomenclature $^{7-10}$ to the etiological theories ${ }^{7-15}$ to the resultant treatment modalities. ${ }^{8-17}$ These controversies have been critically reviewed by DeBoever. ${ }^{18}$

What is lacking in most of these studies has been even ordinal scale quantification of the physiological response of the patients. Results

Supported by USPHS Grant DE-02731.

Received for Publication October 11, 1976

Accepted for publication December 6, 1976. based on the subjective verbal report of the patients are subject to bias and are difficult, if not impossible, to objectively evaluate.

The purpose of the present research was to compare two reportedly quantitative diagnostic techniques ${ }^{1,3}$ measured in the same subjects at the same session. A preliminary version of this research has been presented as an abstract. ${ }^{19}$

\section{Materials and Methods}

The material for this study comprised nine normal subjects and ten patients with TMJmuscle-pain dysfunction from the clinic of the Department of Occlusion, University of Michigan School of Dentistry.

All the patients had pain in the joint or muscles of mastication or both, tenderness to palpation of muscles or joints or both, limited mandibular movement, or joint sounds such as clicking and in some instances, crepitus. To be considered normal, a subject could not have any symptoms of TMJ-muscle-pain dysfunction nor any history of such symptoms.

The clinical protocol for each subject or patient was as follows. Four separate sessions were recorded, one prior to treatment; a second, usually two weeks later, after delivery of a full arch maxillary occlusal splint; a third, four to six weeks later, at cessation of symptoms; and a fourth session six months following the first. The normal subjects followed a similar schedule but received no treatment. Only the initial data are reported here, with the addition of the final data for successfully treated patients. Treatment was considered successful after patient report and clinical examination revealed absence of original symptoms for a period exceeding one month.

Within each session, data were recorded during a standardized sequence of events including rest, maximum clench, chewing, taps to the menton during maximum clench, and the open-close-clench cycle. Only the silent periods from the taps during maximum clench and the 
jaw motion during the closing portion of the open-close-clench are reported here.

The electromyographic methods included recording from the masseter and anterior temporal muscles bilaterally with bipolar surface electrodes. After amplification, the direct traces from the four muscles were simultaneously recorded on FM magnetic tape at 30 inches per second. Upon replay at 7.5 inches per second for time expansion, the silent period durations were measured. To provide consistency of measurement, the silent period was measured from the last peak of the preceding activity to the first peak of the ongoing activity after the period of inhibition.

At the same time as the electrodes were placed, a small permanent magnet was cemented to a lower incisor and a magnetic field sensing device to an upper incisor. This signal was amplified, displayed on the polygraph, and stored on magnetic tape. ${ }^{20}$ Computer processing, described in detail in another publication, ${ }^{3}$ provided a plot of jaw position versus jaw closing velocity and the error between the experimental data and a mathematical model. The mathematical model was a parabola derived from a least-square-error fit to the experimental data. The error between the experimental data and this parabola is referred to as "jaw motion error" or as "phase plane error."

The statistical treatment of the data involved computing the Spearman rank correlation coefficient ${ }^{21}$ between the silent period duration and the phase plane error. One pair of numbers were available from each of the normal subjects and refractory patients. The other patients each had two pairs of numbers, one pair pretreatment and one pair posttreatment. These latter two sets cannot be considered as independent, however, since two pairs came from each patient. The correlation coefficient was computed using only the pretreatment pair from each subject.

\section{Results}

Typical electromyographic tracings from which silent period durations were measured are shown in Fig 1. The silent period durations for the normal group $(\mathrm{N}=9$ ) ranged from 22 to 34 with a mean of 27.4 milliseconds $(\mathrm{ms})$. For the dysfunction patients $(\mathrm{N}=7)$ who subsequently were successfully treated, the duration ranged from 40 to 76 with an average of $52.3 \mathrm{~ms}$ prior to treatment. After successful treatment by occlusal techniques, ${ }^{15}$ the average silent period

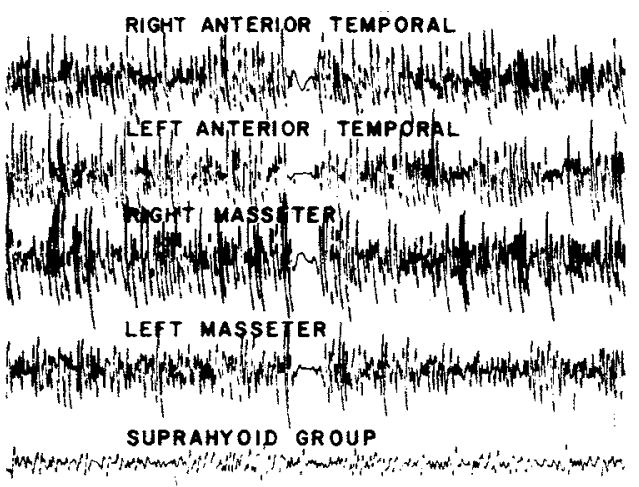

EMG SILENT PERIOD AFTER MENTON TAP

Fig 1.--Typical silent periods. The top five traces show the electrical activity of the labeled muscles. The bottom trace is a timing marker, $250 \mathrm{~ms}$ between marks.

duration for the group dropped to 33.7. The range after treatment was from 27 to $40 \mathrm{~ms}$.

Three dysfunction patients were refractory to treatment with an occlusal splint. Before treatment, the duration of the silent periods of these patients were 18,23 , and 24 ms.

Typical raw data from which phase plane trajectories were computed are shown in Fig 2 The trajectories derived from such traces and the parabolic model are shown in Fig 3. The error between the parabolic model and the experimental data ranged from 10 to 20 with a mean of $14.0 \%$ in the normal subjects. For the dysfunction patients who responded successfully to treatment, the range of error was 20 to 31 with a mean of $24.4 \%$. The previously mentioned refractory patients had pretreatment errors of 10,17 , and $10 \%$.

The purpose of the present research was to see if these two parameters, silent period duration and phase plane error, which differ widely in both neuromuscular mechanism and experimental protocol, supported each other as indicators of clinical dysfunction. The correlation cocfficient was found to be 0.91 which is statistically significant at the 0.01 level. A scatter plot of these data is shown in Fig 4.

While the scatter plot shows four groups (normal, refractory, pretreatment, and post- 

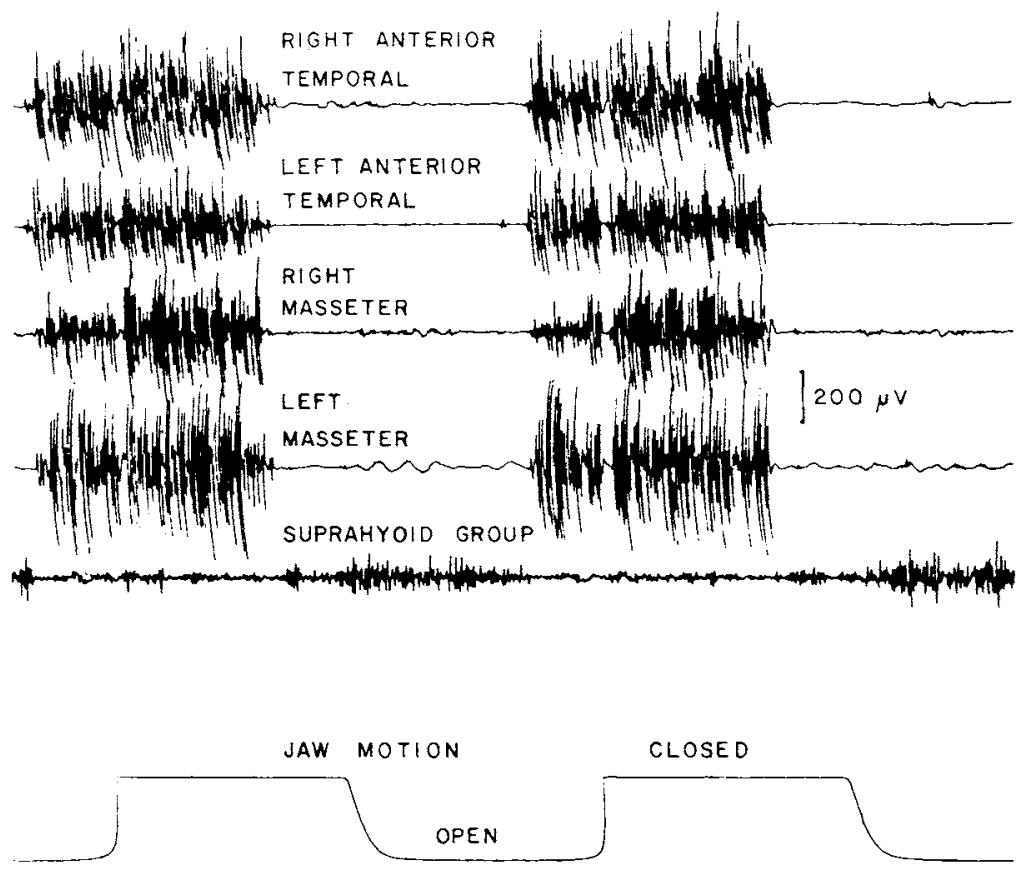

$250 \mathrm{~ms}$

FIG 2.-Typical open-close-clench data. Both the EMG (top five traces) and the jaw motion traces are shown. Due to the nonlinearity of the magnetic field, the jaw motion trace is uncalibrated here. Only the jaw motion during the snap close was analyzed.

treatment), the pretreatment and posttreatment data are from the same patients and therefore are not independent. Accordingly, the correlation coefficient was computed using only the normal, refractory, and pretreatment groups.

\section{Discussion}

The present results and correlations may be discussed both from the viewpoint of neuromuscular mechanisms and from the viewpoint of clinical utility.

Numerous neuromuscular mechanisms have been shown to produce silent periods in animals and man. In the cat, both disfacilitation of primary muscle spindles and autogenic inhibition via Golgi tendon organs ${ }^{22}$ have been shown to be sufficient to produce silent periods under certain experimental conditions. Other possibilities include recurrent inhibition via $\mathrm{R} \leftarrow$ nshaw cells and inhibitory influences from joint receptors.

In man, additional mechanisms have been investigated. Silent periods have been elicited by clectrical stimulation of receptors of the skin or oral mucosa ${ }^{23}$ and by receptors in the periodontal ligament. ${ }^{24,25}$ Those experiments, taken together, indicate a rich and multifarious collection of mechanisms to be investigated. Widmalm ${ }^{2}$ outlines the various possible neuromuscular mechanisms.

On the clinical side, previous reports on silent period durations $1,2,26$ have suggested that they at least segregate normal subjects from dysfunctional patients. Other abstracts, ${ }^{27,28}$ theses, ${ }^{29}$ and personal communications (W. DeReijk and G. Jones, U. Nebraska) support these results.

In addition to these positive clinical results, there is one apparently negative report. An Australian group reported no difference in silent period duration between a group of normal subjects and a group of dysfunction patients. ${ }^{30}$ These investigators, however, elicted the silent periods with the mandible at rest rather than at maximal clench. This important difference in method appears to be sufficient to explain the difference in results. 


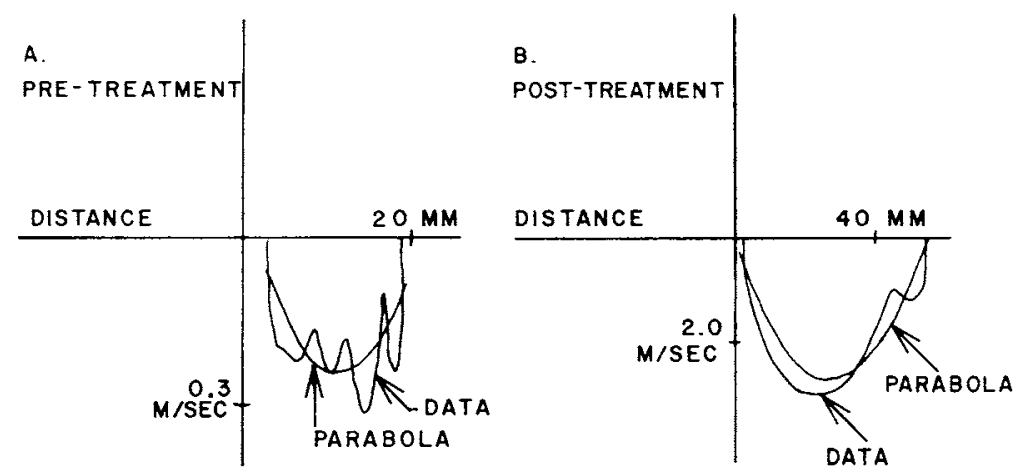

Fig 3.-Typical phase trajectories. A, Dysfunctional patient prior to treatment. B, The same patient subsequent to successful treatment by occlusal techniques. The error between the pre-treatment experimental data and the parabolic model was $24 \%$ prior to treatment and $15 \%$ after treatment.

The clinical significance of the results of this study is that this spectrum of subjects, which may be identified subjectively and clinically by symptoms or lack of symptoms, may also be identified objectively and physiologically by either their silent period durations or their phase plane errors. These two parameters correlate well with each other. What is not intended, and what correlation coefficients fail to demonstrate, is cause and effect. These parameters correlate so well, one might speculate, be- cause they in some presently unknown way "measure" or correlate with the cause of the dysfunction. Thus, even in the absence of more certain knowledge about the etiology, these two parameters tend to support each other as indicators of dysfunction.

It was not anticipated that two such different experiments, one static and one dynamic, one reflex and one voluntary, one electromyographic and one kinesiographic, would provide results that correlate well while purporting to

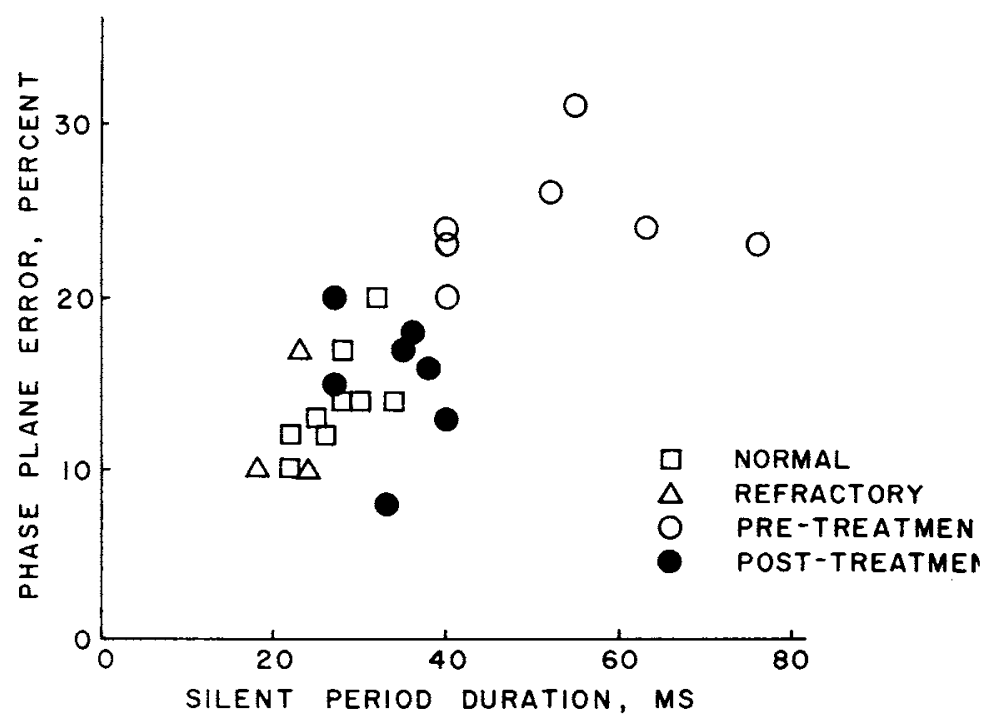

Fig 4.--Scatter plot. The phase plane error and silent period duration for each subject and patient are shown. The results from the posttreatment patients are included for information only and were not used to compute the correlation coefficient. The Spearman rank correlation coefficient was 0.91 ; this is statistically significant at the 0.01 level. 
"measure" the same clinical entity. These two methods of quantification thus reinforce each other and the significant correlation appears to enhance the credence of each for evaluating TMJ-muscle-pain dysfunction.

\section{References}

1. Bessette, R.; Bishop, B.; and Mohr, N.: Duration of Masseteric Silent Period in $\mathrm{Pa}-$ tients with TMJ Syndrome, $J$ Appl Physiol 30:864-869, 1971.

2. Widmalm, S.E.: The Silent Period in the Masseter Muscle of Patients with TMJ Dysfunction, Acta Odont Scand 34:43-52, 1976.

3. MaGall, W.D., JR.; Bailey, J.O., JR.; and Asm, M.M., JR: A Quantitative Measure of TMJ Dysfunction: Phase Plane Modeling of Jaw Motion in Man, Arch Oral Biol, Accepted for publication, 1976.

4. Agerberg, G., and Garlsson, G.E.: Functional Disorders of the Masticatory System: I. Distribution of Symptoms According to Age and Sex as Judged from Investigation by Questionnaire, Acta Odont Scand 30:579$613,1972$.

5. Solberg, W.K.; Woo, M.W.; and HousTON, J.B.: Prevalence of Signs and Symptoms of Mandibular Dysfunction, Proc 1975 $I A D R$, P. 153 (abs.), 1975.

6. Bush, F.M.; AвgotT, D.M.; and Butrer, J.H.: A Population Survey of Jaw Dysfunction, Proc 1976 IADR, P. B311 (abs.), 1976.

7. Schwartz, L.L.: A Temporomandibular Joint Pain-Dysfunction Syndrome, J Chron Dis 3:284-293, 1956.

8. Greene, C.S., and Laskin, D.M.: Splint Therapy for the Myofacial Pain-Dysfunction Syndrome: A Comparative Study, JADA 84: 624-628, 1972.

9. Molin, C.: Studies in Mandibular Pain Dysfunction Syndrome, Swed Dent J (suppl 4) $66: 1-55,1973$.

10. Campbell, J.: Distribution and Treatment of Pain in Temporomandibular Arthroses, Brit Dent J 105:393-402, 1958.

11. Weinberg, L.A.: Correlation of Temporomandibular Dysfunction with Radiographic Findings, J Pros Dent 28:519-539, 1972.

12. KYDD, W.L.: Psychosomatic Aspects of Temporomandibular Joint Dysfunction, JADA 59:31-44, 1959.

13. Lupton, D.E.: Psychological Aspects of Temporomandibular Joint Dysfunction, JADA 79:131-136, 1969.

14. Travell, J.: Temporomandibular Joint Pain Referred from Muscles of the Head and Neck, J Pros Dent 10:745-763, 1960.

15. RAMF JORD, S.P., and Ash, M.M., Jr.: $O c$ clusion, 2nd ed., Philadelphia, Pa., Saunders, 1971.
16. Schwartz, L.L., and Tausig, D.P.: Temporomandibular Joint Pain-Treatment with Intramuscular Infiltration of Tetracaine Hydrochloride: A Preliminary Report, $N Y$ Dent J 20:219-223, 1954.

17. Weinberg, L.L.: Temporomandibular Dysfunction Profile: A Patient-Oriented Approach, J Pros Dent 32:312-325, 1974.

18. DeBoever, J.A.: Functional Disturbances of the Temporomandibular Joints, Oral Sci Rev 2:100-117, 1973 .

19. MaCall, W.D., JR.; Bailey, J.O., JR.; and Asi, M.M., JR.: Electromyographic Silent Periods and Jaw Motion Parameters: Quantitative Measures of Temporomandibular Joint Dysfunction, Fifth Inter Cong EMG $5: 44,1975$.

20. MaCall, W.D., JR., and Rohan, E.J.: A Linear Position Transducer Using a Magnet and Hall Effect Devices, Submitted for publication, 1976.

21. Siegal, S.: Nonparametric Statistics for the Behavioral Sciences, McGraw Hill, New York, 1956.

22. Granit, R.: The Basis of Motor Control, Academic Press, New York, 1970.

23. Yu, S.K.J.; Schmitt, A.; and Sessle, B.J.: Inhibitory Effects on Jaw Muscle Activity of Innocuous and Noxious Stimulation of Facial and Intraoral Sites in Man, Arch Oral Biol 18: 861-870, 1973.

24. Beaudreau, D.E.; Daugherty, W.F.; and Masland, W.S.: Two Types of Motor Pause in Masticatory Muscles, Am J Physiol 216: $16-21,1969$.

25. Sessle, B.J., and Sghmitt, A.: Effects of Gontrolled Tooth Stimulation on Jaw Muscle Activity in Man, Arch Oral Biol 17:15971607, 1972.

26. Beemsterboer, P.L.; MaNamara， D.C.; Holden, S.; and Asm, M.M., JR.: The Effect of Bite Plane Splint on the Electromyographic Silent Period Duration, J Oral Rehab Accepted for publication, 1976.

27. MaNamara, D.C.; Crane, P.F.; MaCall, W.D., JR.; and Ash, M.M., JR.: Myotatic Reflex Response to Unloading and Loading Jaw-Jerk Stimulation, Proc IADR, P. 205 (abs.), 1974.

28. Skiba, T.J., and Laskin, D.M.: Masticatory Muscle Silent Periods in Patients with MPD Syndrome, Proc. IADR, 54:B249 (abs.) , 1976.

29. BAILEY, J.O., JR.: The Electromyographic Silent Period in Normal Subjects and $\mathrm{Pa}$ tients with TMJ-Muscle-Pain Dysfunction, M.S. Thesis, University of Michigan, 1976.

30. MunRo, R., and Griffin, C.J.: Electromyography of the Jaw Jerk Recorded from the Masseter and Anterior Temporalis Muscles in Man, Arch Oral Biol 16:59-69, 1971. 